\title{
Structural Organization of the Cuticle of Nematodes
}

\author{
W. de Souza ${ }^{*}$, A.H.A. de Moraes Neto*, R.M. Lanfredi", and C. Achete ${ }^{* *}$. \\ *Instituto de Biofísica-UFRJ, CCS-Bloco G, Ilha do Fundão, Rio de Janeiro, Brasil \\ ** Department of Mettalurgy, COPPE-UFRJ, Rio de Janeiro, Brasil
}

Nematodes comprise a large number of organisms, including some that are pathogenic for plants and animals. Their external surface is characterized by the presence of a cuticle, a structure that can be considered as an extracellular matrix synthesized by the epidermal cells. The fine structure of the cuticle of adult forms and microfilariae of the filariid Litomosoides chagasfilhoi has been analyzed based on observations made by transmission electron microscopy (TEM) and specially in deep-etched replicas of fully developed intrauterine microfilariae and mature stretched microfilariae released by adult females through cultivation in vitro.

The cuticle of this nematode consists of a trilaminate epicuticle, the outermost layer that interfaces with the host, and our other layers: cortical, intermediate, fibrous and basal (Fig. 1). In deep-etched replicas, the cortical layer was formed by a meshwork of globular particles and fibers with a thickness of 4-8 nm (Fig. 2). The intermediate layer was electron lucid and contained a densely-stained line. In deep-etched replicas it was composed by a meshwork of fibers with a longitudinal orientation. The fibrous layer was the thickest and most electrondense, and consisted of two types of fibers: thick (9-26 nm) and thin (3-6 nm). The innermost basal layer was intimately associated with the hypodermis. In common with other nematodes, the cuticle of $L$. chagasfilhoi presented channels that are probably involved in nutrient acquisition and transport (Fig. 3). In order to obtain further information on the structural organization of the cuticle, it was isolated from adult forms. The purity of the fraction obtained was determined by light and transmission electron microscopy, and the structure of the isolated cuticle studied using deep-etching, high resolution scanning electron microscopy, atomic force microscopy, immunocytochemistry, gel electrophoresis and Western-blot. The epicuticle presented a rugous surface, with parallel rows and several globular particles that probably can be involved in the absorption of nutrients and secretion ofproducts (Fig. 4). SDS-PAGE revealed five major polypeptides corresponding to 151, 41, 28,13 and $11 \mathrm{kDa}$. A polyclonal antibody against a synthetic 18 aminoacid peptide, that corresponds to the sequence ofdomain E ofthe Haemonchus contortus 3A3 collagene gene, recognized several protein bands on Western blot of purified cuticles and labeled all cuticular layers, as shown by immunocytochemistry.

We also analyzed the sheath of microfilariae of L. chagasfilhoi. Transmission electron microscopy showed that the sheath was trilaminated (Fig. 5). In contrast, in deep etching replicas the sheath presented two layers (Fig. 6): an inner layer composed of tightly arranged globular material, and an outer layer whose external surface was relatively smooth. 


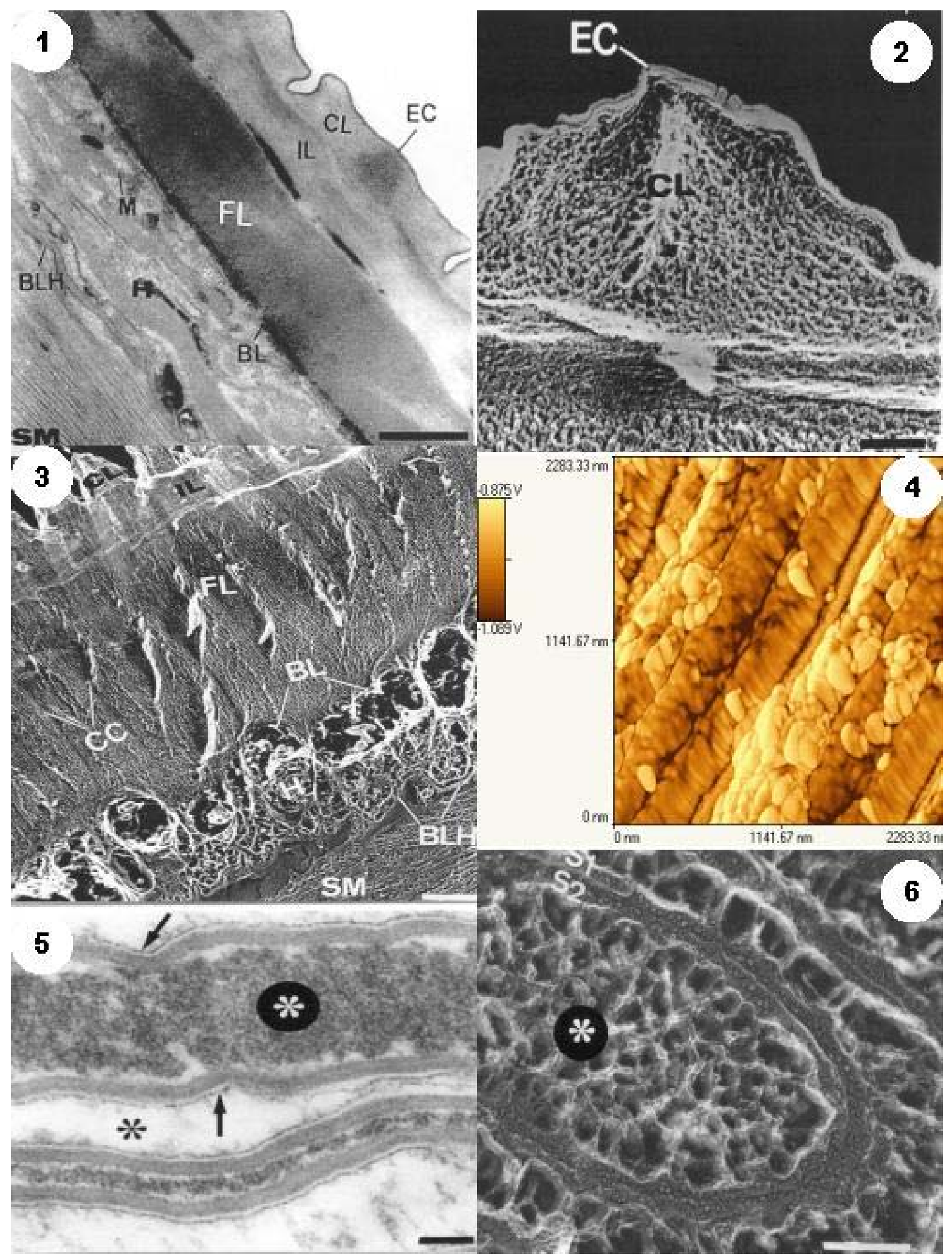

